Supplementary Information

\title{
Influence of chemical and enzymatic TEMPO- mediated oxidation on chemical structure and nanofibrillation of lignocellulose
}

Jie Jiang ${ }^{\text {a, \#, Huangjingyi Chen }}{ }^{\text {a, }, \text { Liang Liu }}{ }^{\text {a }}$, Juan Yu ${ }^{\text {a }}$, Yimin Fan ${ }^{\mathrm{a}, *}$, Tsuguyuki Saito ${ }^{\mathrm{b}}$ and Akira Isogai ${ }^{b, *}$

a Jiangsu Co-Innovation Center of Efficient Processing and Utilization of Forest Resources, Jiangsu Key Lab of Biomass-based Green Fuel \& Chemicals, Key Laboratory of Forestry Genetics \& Biotechnology of Ministry of Education, College of Chemical Engineering, Nanjing Forestry University, No.159 Longpan Road, Nanjing 210037, China

${ }^{b}$ Department of Biomaterial Science, Graduate School of Agricultural and Life Sciences, University of Tokyo, 7-3-1 Hongo, Tokyo 113-8657, Japan

\# The authors contribute equal to the manuscript.

*Corresponding author, E-mail:

*Yimin Fan: fanyimin@njfu.edu.cn;

*Akira Isogai: aisogai@mail.ecc.u-tokyo.ac.jp

The number of pages: 4 pages.

The number of figures: 3 figures.

The number of tables: 2 tables 
Table S1. Chemical composition of CLs.

\begin{tabular}{cccc}
\hline Samples & Cellulose (\%) & Hemicelluloses (\%) & Lignin (\%) \\
\hline CL1 & 71.5 & 18.2 & 5.9 \\
CL2 & 72.5 & 18.7 & 3.8 \\
CL3 & 73.8 & 18.9 & 2.1 \\
CL4 & 74.3 & 19.1 & 1.7 \\
\hline
\end{tabular}

Table S2. Lignin contents, carboxylate contents, and water retention values of oxidized TMPs prepared under various conditions.

\begin{tabular}{lccc}
\hline Sample & $\begin{array}{c}\text { Carboxylate } \\
\text { content }(\mathrm{mmol} / \mathrm{g})\end{array}$ & $\begin{array}{c}\text { Lignin content (\%) } \\
\text { TMP }\end{array}$ & $\begin{array}{c}\text { Water retention } \\
\text { value }(\%)\end{array}$ \\
TNN-TMP-5 mmol/g & 1.04 & 20.2 & 76.9 \\
TNN-TMP-10 mmol/g & 1.65 & 17.5 & 148.6 \\
TNN-TMP-15 mmol/g & 1.72 & 16.1 & 369.1 \\
TNN-TMP-20 mmol/g & 1.58 & 9.8 & 968.9 \\
TNN-TMP-25 mmol/g & 1.57 & 5.7 & 1175 \\
TLO-TMP-1 time & 0.82 & 5.1 & 1537 \\
TLO-TMP-2 times & 1.21 & 19.5 & 114.1 \\
TLO-TMP-3 times & 1.36 & 19.1 & 201.4 \\
TLO-TMP-4 times & 1.43 & 18.3 & 325.7 \\
TLO-TMP-5 times & 1.54 & 17.7 & 1129 \\
TLO-TMP-6 times & 1.82 & 17.1 & 1231 \\
\hline
\end{tabular}




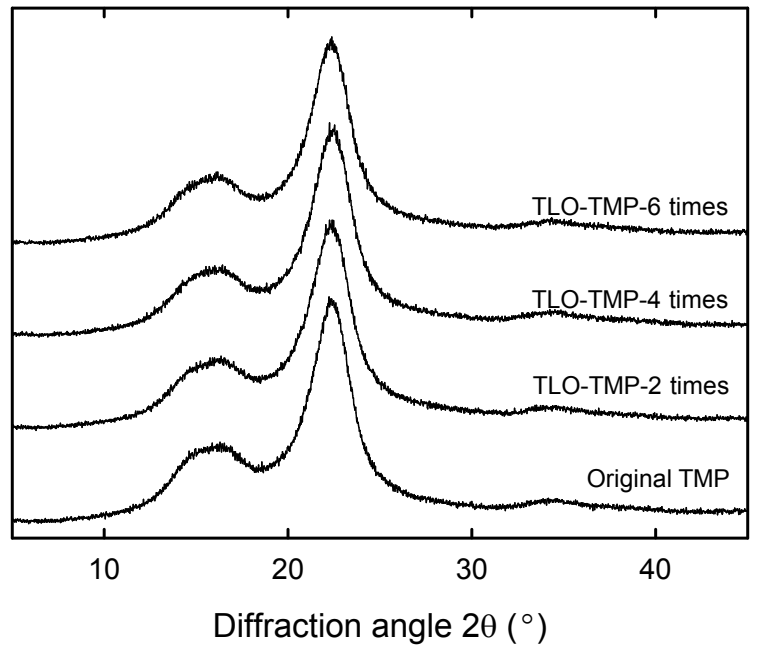

Fig. S1. XRD patterns of TLO-TMPs.
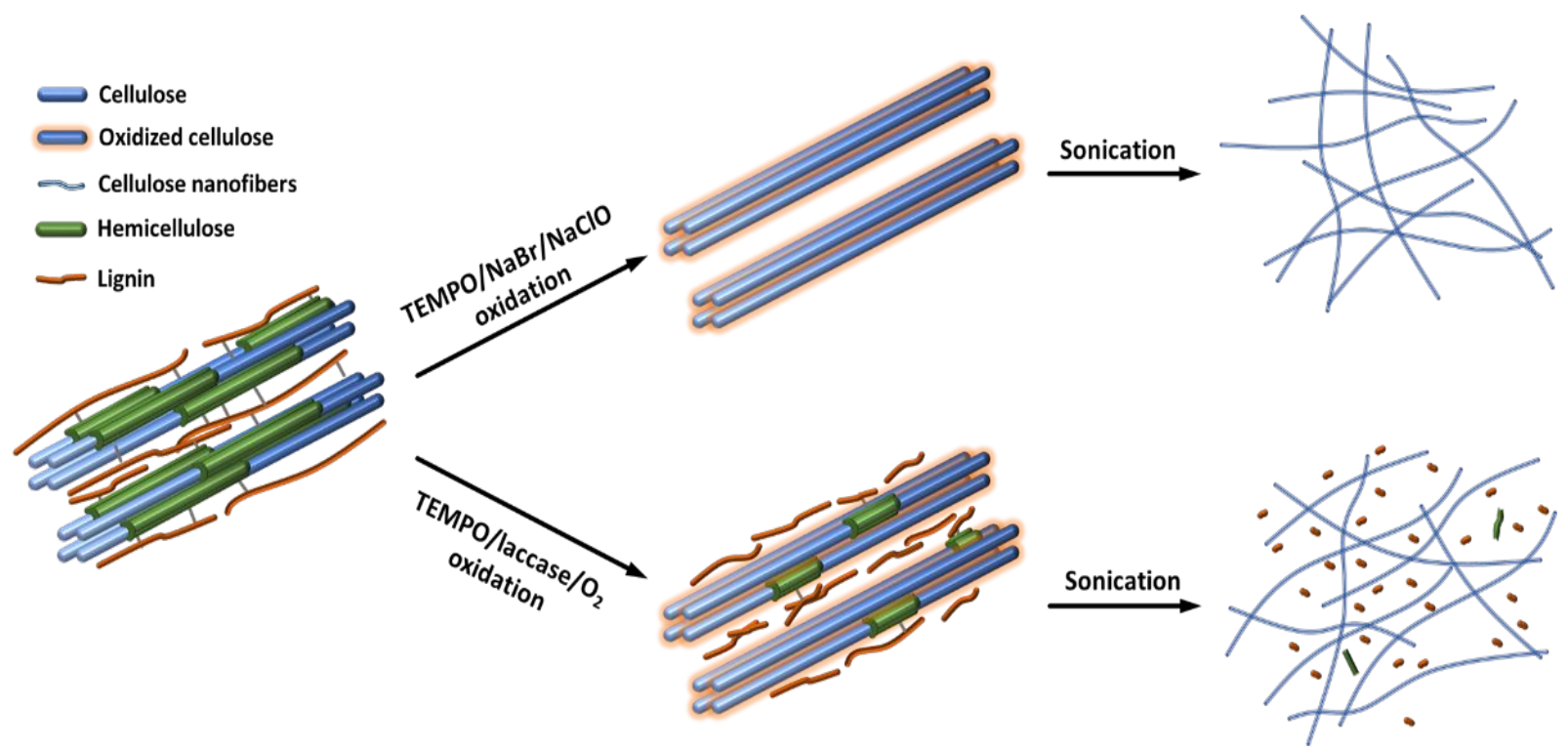

Fig. S2. Possible structural changes of TMP during TEMPO/NaBr/ $\mathrm{NaClO}$ and TEMPO/laccase $/ \mathrm{O}_{2}$ oxidations and the subsequent sonication in water. 


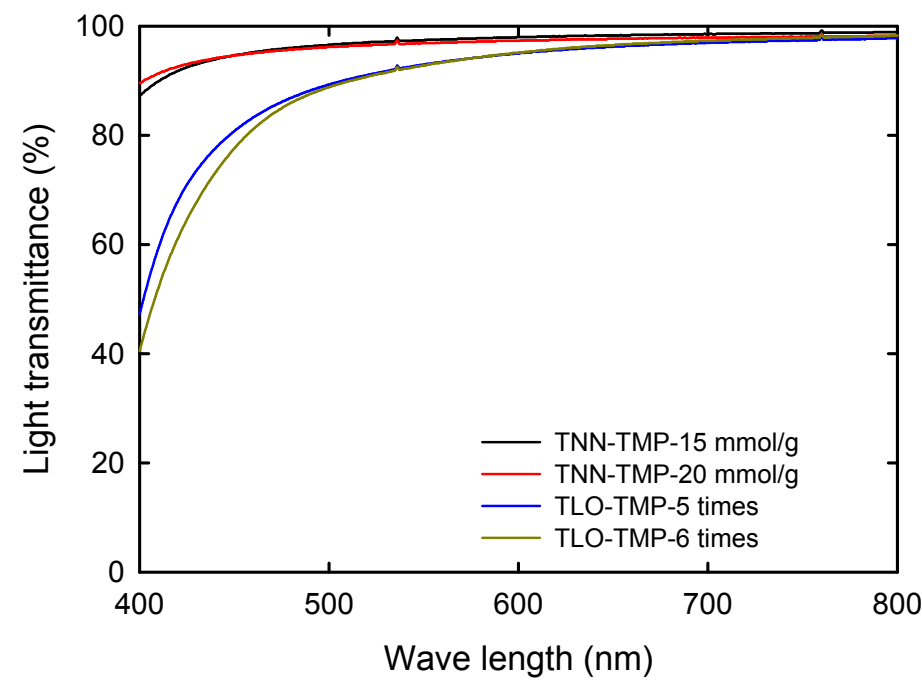

Fig. S3. Light transmittance spectra of $0.1 \%$ aqueous lignocellulose dispersion prepared from TNN-TMP and TLO-TMP under different conditions. 